\title{
Assessment of myocardial perfusion and contractile function by inotropic stress Tc-99m sestamibi SPECT imaging and echocardiography for optimal detection of multivessel coronary artery disease
}

R S Khattar, R Senior, A Lahiri

\begin{abstract}
Objective-To assess whether inotropic stress myocardial perfusion imaging, echocardiography, or a combination of the two could enhance the detection of multivessel disease, over and above clinical and exercise electrocardiographic data.

Design-100 consecutive patients investigated by exercise electrocardiography and diagnostic coronary arteriography underwent simultaneous inotropic stress Tc99m sestamibi SPECT (MIBI) imaging and echocardiography. MIBI imaging and echocardiographic data were analysed using a 12 segment left ventricular model, and each segment was ascribed to a particular coronary artery territory. The presence of perfusion defects with MIBI imaging or of wall thickening abnormality with echocardiography in at least two coronary artery territories at peak stress was taken as diagnostic of multivessel disease. Arteriographic evidence of $\geqslant 50 \%$ stenosis was considered significant.
\end{abstract}

Results-56 patients had multivessel disease. The sensitivity of the combination of MIBI imaging and echocardiography for detecting this was greater than either MIBI imaging or echocardiography alone $(82 \%, 68 \%$, and $68 \%$, respectively; $\mathbf{p}=0.005)$. Clinical and exercise electrocardiographic variables gave an $R^{2}$ value of $18.2 \%$ for predicting multivessel disease. The addition of either MIBI imaging $\left(R^{2}=29.2 \% ; p=0.002\right)$ or echocardiography $\left(R^{2}=28.8 \% ; p<0.001\right)$ enhanced the detection of multivessel disease, and the inclusion of both had further incremental value $\left(R^{2}=34.8 \% ; \quad p=0.003\right)$. Age $(p=0.03)$, MIBI imaging $(p=0.007)$, and echocardiography $(p=0.001)$ were independent predictors of multivessel disease. Conclusions-The assessment of both myocardial perfusion and contractile function by simultaneous inotropic stress MIBI imaging and echocardiography optimises the non-invasive detection of multivessel disease.

(Heart 1998;79:274-280)

Keywords: multivessel disease; inotropic stress; SPECT imaging; echocardiography
Flow heterogeneity and systolic wall thickening abnormality are two of the earliest manifestations of myocardial ischaemia. ${ }^{1}$ The detection of flow heterogeneity forms the conceptual basis for myocardial perfusion imaging, and systolic wall thickening abnormality is readily detected by echocardiography. As these manifestations occur before the onset of symptoms and electrocardiographic changes, it is not surprising that many studies have shown that exercise and pharmacological stress testing, in conjunction with myocardial perfusion imaging or echocardiography, is superior to electrocardiography for detecting coronary artery disease. ${ }^{2-4}$ Although these stress imaging modes show good diagnostic accuracy for the overall detection of coronary artery disease, their ability to identify multivessel involvement correctly is often suboptimal. ${ }^{5}$ The correct detection of multivessel coronary artery disease is important because it is in this category of patients that revascularisation procedures may improve outcome. ${ }^{6-8}$ Whether the combination of stress myocardial perfusion imaging and echocardiography can enhance the detection of multivessel disease in conjunction with clinical and exercise electrocardiographic variables has not previously been investigated. Inotropic stress testing (using dobutamine or arbutamine) is a safe, accurate, and increasingly used technique for evaluating coronary artery disease, ${ }^{9-14}$ and whether it is used in conjunction with myocardial perfusion imaging or echocardiography, its diagnostic efficacy is similar to dynamic exercise. ${ }^{15-17}$ The technique has the added advantage of allowing assessment in patients who are unable to exercise. The aim of our study was to determine whether combining inotropic stress testing with myocardial perfusion imaging, echocardiography, or both, can provide more information than clinical variables and exercise electrocardiography alone in the detection of multivessel disease.

\section{Methods}

PATIENTS

The study group consisted of consecutive patients with chest pain undergoing exercise electrocardiography and subsequent coronary arteriography for diagnostic purposes. Specific exclusion criteria included unstable angina, myocardial infarction within 30 days, significant arrhythmias, heart failure, cardiomyopathy, significant valvar disease, and uncontrolled
Accepted for publication 3 November 1997 
hypertension. $\beta$ Blockers and heart rate lowering calcium antagonists were withdrawn 48 hours before stress testing. The study protocol was approved by the hospital ethics committee and all patients gave informed consent before participating in the study.

EXERCISE TESTING

A Bruce protocol, symptom limited treadmill test was performed using a microcomputer based system (Case 12, Marquette Electronics, Milwaukee, Wisconsin, USA) linked to a motor driven treadmill. The electrocardiogram was continuously monitored during exercise and for a minimum of five minutes during the recovery phase; blood pressure was measured at rest and at each stage of exercise. ST segment depression at J point $+60 \mathrm{~ms}$ was computed and the metabolic equivalents automatically calculated. The exercise test was terminated in the presence of horizontal or downsloping ST depression $\geqslant 3 \mathrm{~mm}$ below the resting level in at least two contiguous leads. The test was considered positive for the detection of multivessel disease in the presence of either ST depression $\geqslant 2 \mathrm{~mm}, \mathrm{ST}$ depression $\geqslant 1 \mathrm{~mm}$ involving five leads or more, workload $<6$ metabolic equivalents, or significant hypotension defined as a fall of systolic blood pressure $>20 \mathrm{~mm} \mathrm{Hg}$ compared to the previous stage. ${ }^{18}$

INOTROPIC STRESS IMAGING PROTOCOL

Inotropic stress testing was performed using either dobutamine or arbutamine. Dobutamine was infused through a peripheral intravenous line starting at a dose of $5 \mu \mathrm{g} / \mathrm{kg} / \mathrm{min}$ followed by $5 \mu \mathrm{g} / \mathrm{kg} / \mathrm{min}$ increments every three minutes to a maximum dose of $40 \mu \mathrm{g} / \mathrm{kg} / \mathrm{min}$ if required. Arbutamine infusion was facilitated by a computerised closed loop delivery device that continuously monitored heart rate and measured blood pressure at two minute intervals. The rate of arbutamine infusion was controlled according to a predetermined heart rate slope of 4 beats $/ \mathrm{min}$ and a maximum heart rate target of $85 \%$ of the age predicted maximum. End points included intolerable symptoms, target heart rate, extensive wall motion abnormality, significant arrhythmia, heart rate saturation (plateau heart rate response despite increasing doses of inotropic stress agent to a maximum total dose of $40 \mu \mathrm{g} /$ $\mathrm{kg} / \mathrm{min}$ for dobutamine or $10 \mu \mathrm{g} / \mathrm{kg}$ for arbutamine), or maximum dose. Echocardiography was performed at baseline, at low inotropic dose $(10-15 \mu \mathrm{g} / \mathrm{kg} / \mathrm{min}$ of dobutamine and three minutes into arbutamine infusion), at peak stress, and 10 minutes into the recovery period. Tc-99m sestamibi was injected intravenously one minute before termination of the stress test. Scintigraphic images were obtained one to two hours later, and rest images were acquired with the same dose of Tc- $99 \mathrm{~m}$ sestamibi within three to seven days thereafter.

\section{ECHOCARDIOGRAPHY}

Echocardiographic images were obtained in the parasternal long and short axis and apical four and two chamber views, using a $2-3 \mathrm{MHz}$ broad band transducer (ATL, Ultramark HDI $\mathrm{CV}$, Bothell, Washington, USA). Images were stored on VHS videotape and digitised on-line with an $\mathrm{R}$ wave trigger to obtain a continuous loop, quad-screen display with the DCR Image-Vue system (Nova Microsonics, Mahwah, New Jersey, USA). Analysis of the rest and peak stress images was performed from the digitised quad-screen display and from videotape playback. The multiview echocardiographic images of the left ventricular myocardium were extrapolated into a 12 segment model illustrated in fig 1 , as previously described. ${ }^{19}$ Each segment was graded for systolic wall thickening as follows: 1 = normal, $2=$ mildly reduced wall thickening, $3=$ severely reduced wall thickening, $4=$ absence of wall thickening. Coronary artery disease was diagnosed on the basis of a resting or stress induced wall thickening abnormality.

\section{TC-99M SESTAMIBI SPECT IMAGING}

Images were acquired 60 to 120 minutes after the injection of $600 \mathrm{MBq}$ of Tc-99m sestamibi (MIBI) with a large field of view gamma camera using a high resolution collimator. Thirty two projections were made over a $180^{\circ}$ arc, from the $45^{\circ}$ right anterior oblique to the $45^{\circ}$ left posterior oblique position. Rest and stress images in the vertical and horizontal long axis and short axis views were displayed simultaneously and a 12 segment left ventricular model identical to that for echocardiography was used. Each segment was given a semiquantitative score for tracer uptake such that $1=$ normal uptake, $2=$ mildly reduced uptake, $3=$ severely reduced uptake, and $4=$ absent uptake. Coronary artery disease was diagnosed on the basis of a resting or stress induced perfusion defect.

CORONARY ARTERIOGRAPHY

Coronary arteriography was considered the reference standard for the detection of coronary artery disease and was performed within three months of both stress tests. Arteriographic images were obtained in multiple projections by the Judkins technique. Images were recorded on $35 \mathrm{~mm}$ cine film or videotape and evaluated qualitatively by two independent experienced observers who were unaware of the non-invasive imaging results. Evidence of $a \geqslant 50 \%$ diameter stenosis was considered significant, and multivessel disease was defined as the involvement of at least two major coronary arteries.

ANALYSIS

Echocardiographic and MIBI images were analysed at random by two separate pairs of observers blinded to clinical, arteriographic, and relevant imaging data. As shown in fig 1, abnormalities in the five anterior and anteroseptal segments were ascribed to the left anterior descending coronary artery, in the five inferior and inferoseptal segments to the right coronary artery, and in the two lateral segments to the left circumflex coronary artery. The apex was allocated to any of the affected territories, or if the apex alone was involved, disease was 
designated to the left anterior descending coronary artery. The detection of multivessel disease, for each imaging mode, was considered positive if abnormalities were identified in at least two coronary artery territories at peak stress.

STATISTICAL ANALYSIS

The variables analysed included age, gender, previous myocardial infarction, hypertension, diabetes mellitus, and current smoking history, along with exercise electrocardiographic variables and inotropic stress MIBI imaging and echocardiographic criteria for the detection of multivessel disease. Sensitivities, specificities, and diagnostic accuracies of exercise electrocardiography, MIBI imaging, and echocardiography were calculated for the detection of multivessel disease. Diagnostic values for the combination of MIBI imaging and echocardiographic data were also derived by assuming that the tests detected disease when either was positive, and that there was no disease when both tests were normal.

The results for each different test were compared using the McNemar test for paired data. Univariate analysis was performed to determine the individual relations of the above variables to the presence of multivessel disease. A logistic regression model containing clinical variables and positive exercise electrocardiography findings for the prediction of multivessel disease was then fitted. Positive MIBI imaging and echocardiographic tests meeting the predefined criteria for the detection of multivessel disease were systematically added to this model, both alone and in combination; respective $R^{2}$ values for each model were calculated. A series of likelihood ratio tests was then performed to assess whether the addition of these variables significantly improved the prediction of multivessel disease. This form of analysis takes in to account both sensitivities

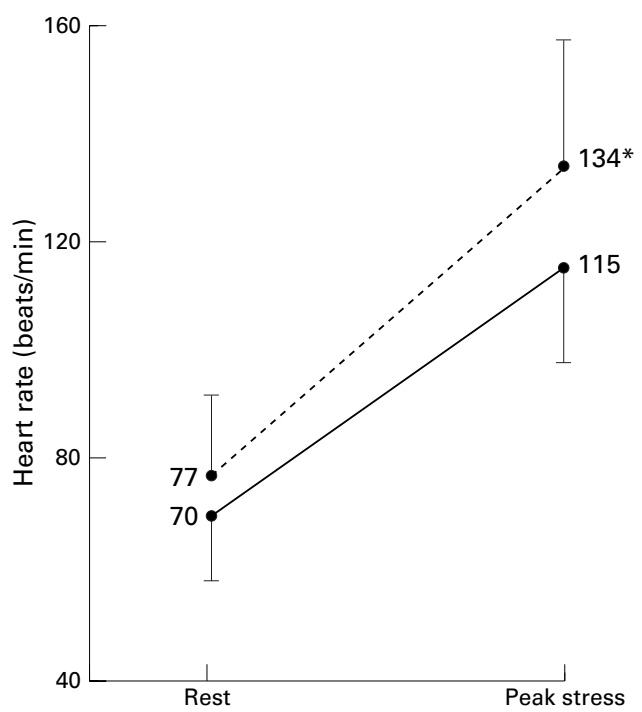

and specificities of the tests in predicting the probability of multivessel disease. Finally, stepwise multiple logistic regression analysis was performed using both forward and backward variable selection to determine the independent predictors of multivessel disease.

Continuous variables were expressed as mean (SD) and categorical variables as the number and percentage of patients so affected. A $p$ value $<0.05$ was considered significant.

\section{Results}

One hundred patients were investigated, of whom 70 were male and 30 female, with a mean age of 62.2 (8.9) years. Thirty four patients gave a positive smoking history, 34 had hypertension, 29 gave a history of previous myocardial infarction, and six were diabetic. Coronary arteriography showed that 56 patients had multivessel disease, of whom 52 had significant left anterior descending coronary artery stenoses, 18 had single vessel disease, and 26 had normal coronary arteries. With respect to inotropic stress testing, 61 patients were given dobutamine and 39 received arbutamine as the stressor agent.

HAEMODYNAMIC CHANGES DURING EXERCISE ELECTROCARDIOGRAPHY AND INOTROPIC STRESS TESTING

The mean duration of symptom limited Bruce protocol treadmill exercise testing was 6.3 (2.8) minutes at an average workload of 7.0 (2.3) metabolic equivalents. As shown in fig 2, the mean increase in heart rate from rest to peak stress was 57.4 (18.5) beats/min for exercise electrocardiography compared to 44.9 (14.9) beats/min for inotropic stress testing $(\mathrm{p}<0.001)$. Systolic blood pressure rose by 26.6 (19.4) $\mathrm{mm} \mathrm{Hg}$ during exercise electrocardiography, compared with 20.1 (26.1) $\mathrm{mm} \mathrm{Hg}$

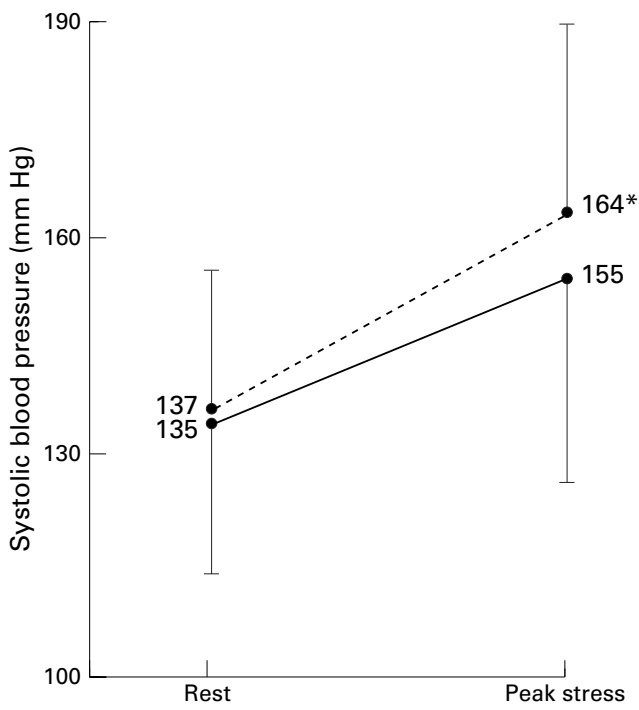



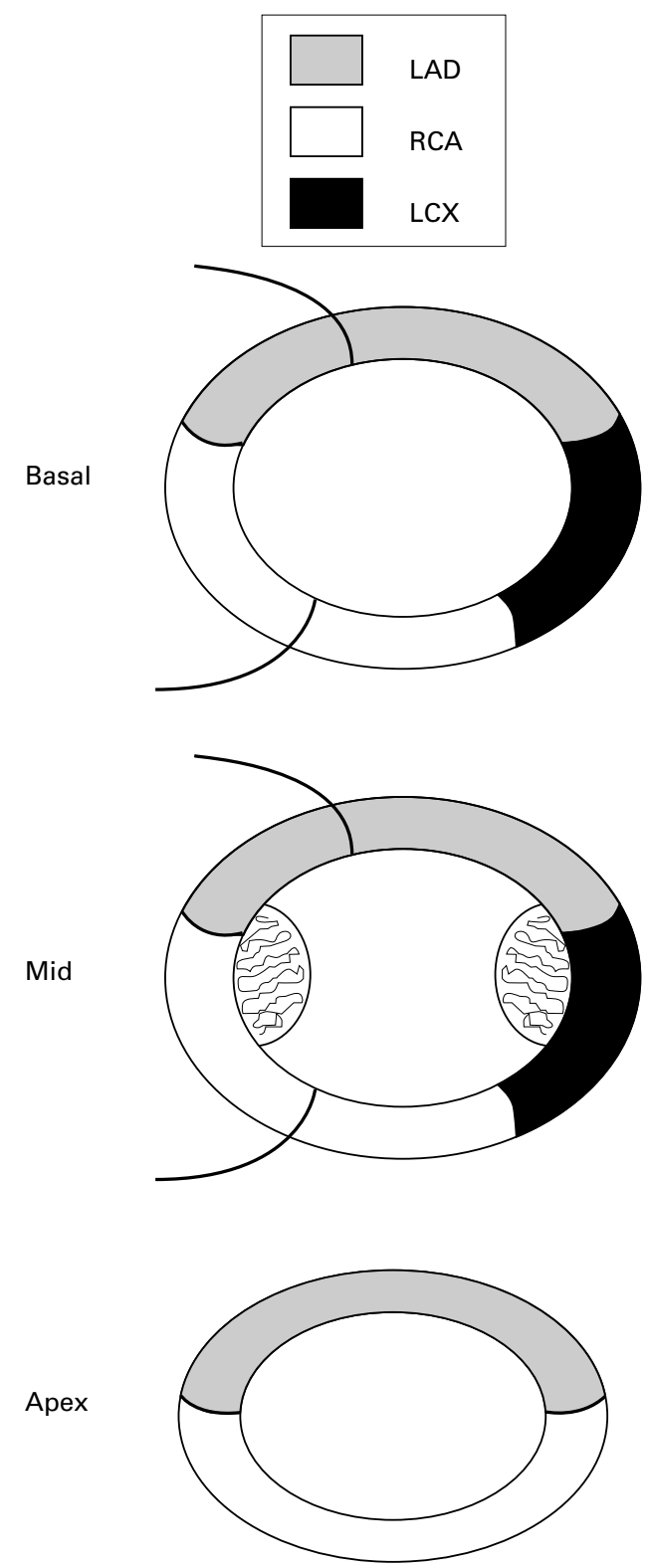

Figure 2 Twelve segments model of the left ventricular myocardium consisting of five segments at the basal level, five at the mid-papillary level, and two at the apex. $L A D$, left anterior descending coronary artery; $R C A$, right coronary artery; $L C X$, left circumflex coronary artery.

for inotropic stress testing $(p<0.001)$. This translated into a significantly higher peak ratepressure product during exercise electrocardiography than during inotropic stress testing: $22.1(5.8) \times 10^{3} v 18.0(4.4) \times 10^{3}$ beats $/ \mathrm{min} /$ $\mathrm{mm} \mathrm{Hg}$, respectively; $\mathrm{p}<0.001$.

REASONS FOR TEST TERMINATION

The primary reasons for termination of exercise electrocardiography and inotropic stress testing are listed in table 1 . Angina was the most common endpoint for exercise electrocardiography, occurring in 49 patients; inotropic stress testing precipitated angina in 23 cases. With respect to the other causes leading to termination of inotropic stress, 29 patients either achieved peak dose or developed heart rate saturation, 18 achieved target heart rate, and seven developed extensive wall thickening
Table 1 Primary end points for termination of stress testing

\begin{tabular}{lrlll}
\hline Exercise ECG & & & \multicolumn{2}{l}{ Inotropic stress } \\
\cline { 1 - 2 } \cline { 5 - 5 } End point & $n$ & & End point & $n$ \\
\hline Angina & 49 & & Angina & 23 \\
Dyspnoea & 22 & & HR sat/peak dose & 29 \\
Fatigue & 14 & & Target HR & 18 \\
ST depression & 5 & & Hypotension & 13 \\
Miscellaneous & 10 & & Miscellaneous & 17 \\
& & & &
\end{tabular}

HR sat, heart rate saturation.

abnormality. Hypotension occurred in 13 cases and five patients developed ventricular arrhythmias (frequent ventricular ectopics in four cases and non-sustained ventricular tachycardia in one); these arrhythmic and hypotensive episodes resolved spontaneously after test termination without the need for specific treatment. Miscellaneous endpoints included palpitations, tremor, and nausea.

Sixty seven of the 100 patients failed to achieve target heart rate, of whom 20 experienced angina as the primary endpoint, 29 developed heart rate saturation or achieved maximum dose, 11 became hypotensive, and seven developed palpitations, tremor, or frequent ventricular ectopics.

DIAGNOSTIC VALUES FOR THE DETECTION OF MULTIVESSEL DISEASE

Diagnostic values for exercise electrocardiography, inotropic stress MIBI imaging, echocardiography, and a combination of the last two in the total study population and in subjects without previous myocardial infarction are given in table 2. Thirty two of the 71 patients without previous myocardial infarction had multivessel disease. Although the diagnostic tests had slightly higher sensitivities and lower specificities in the total study population than in the subjects without previous myocardial infarction, the difference between the two groups was not significant. In both groups, the sensitivities of all three tests were very similar, but the specificities and overall diagnostic accuracies of exercise electrocardiography were significantly lower than those of inotropic stress MIBI imaging and echocardiography. Although the diagnostic accuracies of MIBI imaging and echocardiography-performed alone and in combination-were similar, the combined approach significantly improved the sensitivity for detecting multivessel disease compared with either technique alone, without reducing specificity to a statistically significant level.

\section{UNIVARIATE ANALYSIS}

Univariate analysis was performed by dividing the patients into two groups according to the presence or absence of multivessel disease. Comparison of clinical variables, exercise electrocardiographic and inotropic stress MIBI imaging, and echocardiographic data between these two groups is summarised in table 3. Age and the frequency of previous myocardial infarction were significantly greater in the group with multivessel disease. There was also a trend for patients with multivessel disease to achieve a lower exercise workload during 
Table 2 Diagnostic values of exercise electrocardiography, inotropic stress echocardiography, and MIBI imaging for detecting multivessel disease

\begin{tabular}{|c|c|c|c|c|c|c|}
\hline & \multicolumn{3}{|c|}{ Total study group $(n=100)$} & \multicolumn{3}{|c|}{ Excluding previous $M I(n=71)$} \\
\hline & $\begin{array}{l}\text { Sens } \\
(\%)\end{array}$ & $\begin{array}{l}\text { Spec } \\
(\%)\end{array}$ & $\begin{array}{l}\text { Accuracy } \\
(\%)\end{array}$ & $\begin{array}{l}\text { Sens } \\
(\%)\end{array}$ & $\begin{array}{l}\text { Spec } \\
(\%)\end{array}$ & $\begin{array}{l}\text { Accuracy } \\
(\%)\end{array}$ \\
\hline Exercise ECG & 70 & 41 & $57^{\star}$ & 61 & 38 & $48^{\star}$ \\
\hline MIBI & 68 & $73 \dagger$ & 70 & 66 & $82 \dagger$ & 75 \\
\hline Echo & 68 & $82 \dagger$ & 74 & 63 & $85 \dagger$ & 75 \\
\hline MIBI + echo & $82 \ddagger$ & 61 & 73 & $78 \ddagger$ & 69 & 73 \\
\hline
\end{tabular}

Accuracy, diagnostic accuracy; sens, sensitivity; spec, specificity.

${ }^{\star} \mathrm{p}<0.01 v \mathrm{MIBI}$, echo, and MIBI + echo; $\nmid \mathrm{p}<0.01 v$ exercise ECG; $\ddagger \mathrm{p}<0.01 v \mathrm{MIBI}$ and echo alone.

Table 3 Comparison of clinical, exercise electrocardiographic, and inotropic stress imaging data between subjects with and without multivessel disease (MVD)

\begin{tabular}{llll}
\hline & \multicolumn{2}{c}{ Multivessel disease } & \\
\cline { 2 - 3 } & Absent $(n=44)$ & Present $(n=56)$ & p value \\
\hline Clinical variables & & & \\
$\quad$ Age (years) & $58.4(1.5)$ & $63.9(1.1)$ & 0.004 \\
Previous MI & $5(11 \%)$ & $23(41 \%)$ & 0.001 \\
Hypertension & $15(34 \%)$ & $19(34 \%)$ & NS \\
Diabetes mellitus & $1(3 \%)$ & $5(8 \%)$ & NS \\
Smoking & $14(32 \%)$ & $20(36 \%)$ & NS \\
Exercise testing & & & \\
Exercise workload (METs) & $7.4(0.5)$ & $6.4(0.3)$ & 0.06 \\
Exercise worload < 6 METs & $11(25 \%)$ & $24(42 \%)$ & NS \\
ST depression (mm) & $1.8(0.2)$ & $1.6(0.1)$ & NS \\
ST depression $\geqslant 2$ mm & $18(42 \%)$ & $15(27 \%)$ & NS \\
Rate-pressure product & $24.1(1.0)$ & $20.3(0.7)$ & 0.002 \\
Positive criteria for MVD & $29(67 \%)$ & $38(67 \%)$ & NS \\
Inotropic stress imaging & & & \\
$\quad$ Positive MIBI criteria for MVD & $10(28 \%)$ & $35(73 \%)$ & $<0.001$ \\
Positive echo criteria for MVD & $6(17 \%)$ & $34(71 \%)$ & $<0.001$ \\
\hline
\end{tabular}

Values are $\mathrm{n}(\%)$ or mean (SEM).

METs, metabolic equivalents.
Table 4 Logistic regression models showing incremental value of inotropic stress echocardiography (echo) and MIBI imaging for the detection of multivessel disease in conjunction with clinical variables and exercise electrocardiography (Ex ECG)

\begin{tabular}{lll}
\hline Logistic model & $R^{2}$ value & $p$ value \\
\hline Clinical and Ex ECG alone & $18.2 \%$ & - \\
Clinical and Ex ECG + echo & $28.8 \%$ & $<0.001$ \\
Clinical and Ex ECG + MIBI & $29.2 \%$ & 0.002 \\
Clinical and Ex ECG + MIBI + echo & $34.8 \%$ & 0.003 \\
Clinical and Ex ECG + echo + MIBI & $34.8 \%$ & 0.03 \\
\hline
\end{tabular}

and incremental information for the detection of multivessel disease.

\section{Discussion}

Our study is the first to perform a multivariate analysis to assess the relative abilities of stress myocardial perfusion imaging and echocardiography to detect multivessel disease in conjunction with clinical and exercise electrocardiographic data. The findings showed that inotropic stress Tc-99m sestamibi SPECT imaging and echocardiography had independent and incremental value for detecting multivessel disease. Age was the only other independent predictor of multivessel disease. Previous myocardial infarction and peak ratepressure product during treadmill exercise showed positive univariate relations, but none of the exercise electrocardiographic variables was independently related to multivessel disease in our model. In particular, the degree or presence of ST segment depression did not provide any diagnostic value for detecting multivessel disease- a significant proportion of patients in our study had previous myocardial infarction and hypertension, making interpretation of exercise induced ST segment changes less reliable. ${ }^{20}$ Furthermore, the low specificity of exercise electrocardiography may be partly attributable to post-test referral bias; the decision to perform coronary angiography was guided by clinical information and the results of exercise electrocardiography, thereby increasing the likelihood of false positive results with this test.

A recent meta-analysis of 11 studies using simultaneous exercise or pharmacological stress echocardiography and myocardial perfusion imaging showed cumulative sensitivities of $83 \% v 78 \%$ and specificities of $77 \% v 86 \%$ for myocardial perfusion imaging and echocardiography, respectively, for the overall detection of coronary artery disease. ${ }^{21}$ However, only two of these studies specifically addressed the ability of these techniques to detect the presence of multivessel disease. Galanti et al evaluated 53 patients without previous myocardial infarction or resting wall motion abnormality and found exercise echocardiography to be less sensitive than myocardial perfusion imaging for the detection of multivessel disease. ${ }^{22}$ However, the study included only 13 patients with multivessel disease, and specificities of the two imaging modes were not documented. Marwick et al evaluated 97 patients without previous myocardial infarction, of whom 28 of 59 patients with coronary $0.007)$, and echocardiography $(p=0.001)$ to be the only independent predictors of the presence of multivessel disease. Therefore, both techniques provided significant independent 
artery disease had multivessel disease. ${ }^{5}$ The sensitivity of echocardiography was lower than MIBI imaging for the correct detection of multivessel disease in that study, but diagnostic accuracies taking into account specificity were similar. In contrast, Hecht et al found supine bicycle exercise echocardiography to be more sensitive than treadmill exercise thallium-201 SPECT imaging for the correct identification of the number of coronary arteries affected and triple vessel disease. ${ }^{23}$

In the past many studies evaluating either stress myocardial perfusion imaging or echocardiography have shown the incremental value of these tests in identifying patients with multivessel disease. ${ }^{24-29}$ The number of perfusion abnormalities or the multivessel distribution of perfusion abnormalities has been a consistent independent predictor of multivessel disease during myocardial perfusion imaging. Indeed, Pollock et al validated a model based on age, exercise induced ST segment depression, and thallium-201 imaging by showing its good ability to predict the presence of multivessel disease when applied to a patient population different from the original study group. ${ }^{30}$ Although the available data on stress echocardiography are less abundant, Roger et al have similarly shown exercise induced wall motion abnormality in multiple vascular territories to be an independent predictor of multivessel disease, even after taking into account clinical and exercise electrocardiographic variables. ${ }^{31}$

In contrast to our findings, pathophysiological mechanisms based on the ischaemic cascade might suggest that, because flow heterogeneity precedes the development of systolic wall thickening abnormality, ${ }^{1}$ the assessment of contractile function by echocardiography ought not to provide incremental diagnostic information for detecting the presence and extent of coronary artery disease when combined with myocardial perfusion imaging data. However, our findings are supported by a report by Quinones et al, who used simultaneous exercise thallium-201 SPECT imaging and echocardiography and showed that each test detected an equal number of abnormalities not detected by the other mode, ${ }^{32}$ further suggesting that their combined use improves the evaluation of the extent of coronary artery disease.

Possible reasons for the optimal detection of multivessel disease using this combined imaging approach can be attributed to patient related factors, pathophysiological effects, and technical considerations related to image acquisition and interpretation. Although the sensitivity of myocardial perfusion imaging tends to be greater than echocardiography, this is counterbalanced by its reduced specificity, and both of these important facets of diagnostic efficacy are taken into account in multivariate analysis. A previous study has shown that milder degrees of coronary artery disease and submaximal levels of stress tend to disproportionately lower the sensitivity of echocardiography compared with myocardial perfusion imaging. ${ }^{5}$ Notably, in the present study, 67 patients failed to achieve target heart rate and this may be one of the reasons for the additional value of MIBI imaging. The fact that alterations of regional perfusion precede the development of ischaemia may account for the higher sensitivity of perfusion imaging in these circumstances. Equally, however, the reduced specificity of myocardial perfusion imaging may in part reflect the fact that many perfusion defects which are not associated with haemodynamically significant coronary artery disease merely reflect flow heterogeneity. Myocardial perfusion imaging is also more prone to false positive results as a result of soft tissue attenuation and motion artefacts. Furthermore, echocardiography appears to be more specific than myocardial perfusion imaging in patients with left ventricular hypertrophy or left bundle branch block. With respect to the technical aspects, the diagnostic accuracy of SPECT imaging depends on the computer algorithms used to reconstruct the different tomographic planes, the resolution of the scintigraphic camera, and the experience of the interpreting physician. With current state of the art ultrasound equipment and digital image acquisition techniques, stress echocardiography depends for the most part on the technical expertise of the operator and the experience of the interpreting physician. Perhaps the major weakness of echocardiography is the highly subjective assessment of wall motion abnormalities, as quantitative techniques are not in routine use and endocardial border definition can sometimes be suboptimal.

\section{CLINICAL RELEVANCE}

Our findings suggest that both myocardial perfusion and contractile function should be assessed to optimise the non-invasive detection of multivessel coronary artery disease. These findings herald promise for investigational techniques such as gated SPECT imaging ${ }^{33}$ and intravenous myocardial contrast echocardiography, ${ }^{34}$ which have the intrinsic ability to assess both perfusion and function. Until these techniques are fully evaluated, the use of simultaneous stress myocardial perfusion imaging and echocardiography offers the best option for enhancing the non-invasive detection of multivessel disease. Under circumstances in which simultaneous imaging is not practically feasible, we advocate the application of either of the two non-invasive imaging modes, as each of these techniques significantly enhances the detection of multivessel disease in comparison with clinical variables and exercise electrocardiography alone. The choice of test may depend on the presence of concurrent patient related factors that may undermine the diagnostic efficacy of the given test, and on pragmatic issues such as availability, cost, and local expertise.

STUDY LIMITATIONS

Although we used two different catecholamine based stressor agents in the study, we do not think this will have unduly influenced our results, as each imaging mode was performed simultaneously under the same conditions of 
stress for each individual patient. Finally, myocardial perfusion imaging, echocardiography, and coronary arteriography were all assessed visually. The application of quantitative techniques to thallium-201 SPECT imaging may enhance the diagnostic power of the test, but their ability to do the same with Tc-99m labelled agents is as yet unproven.

Quantitative analysis of echocardiographic data remains hampered by technical difficulties. Although qualitative analysis of coronary arteriographic findings has limitations in determining the significance of coronary artery stenosis, similar problems with quantitative coronary arteriography have also been described. ${ }^{35}$ Furthermore, semiquantitative reporting of perfusion imaging and echocardiographic studies - and the visual assessment of arteriographic data as performed in this study - are widely applied methods and important for the generalisation of the results to routine clinical practice.

This study was supported by a grant from the Northwick Park Hospital Cardiac Research Fund, Gensia Europe Ltd, and DuPont Pharma Europe. We would like to thank Usha Raval for her technical expertise in nuclear imaging and Caroline Dore for her statistical services.

1 Nesto RW, Kowalchuk GJ. The ischaemic cascade: temporal sequence of haemodynamic, electrocardiographic and 1987;59(suppl):23-30C.

2 Gibson R, Watson D. Clinical applications of myocardial perfusion scintigraphy with thallium-201. Prog Cardiol 1983;12:67-112.

3 Kotler TS, Diamond GA. Exercise thallium-201 scintigraphy in the diagnosis and prognosis of coronary artery disease. Ann Intern Med 1990;113:684-702.

4 Hoffmann $\mathrm{R}$, Lethen $\mathrm{H}$, Kleinhans $\mathrm{E}$, et al. Comparative evaluation of bicycle and dobutamine stress echocardiography with perfusion scintigraphy and bicycle electrocardiogram for identification of coronary artery disease. $A m \mathcal{F}$ Cardiol 1993;72:555-9.

5 Marwick T, D'Hondt AM, Baudhuin T, et al. Optimal use of dobutamine stress for the detection and evaluation of coronary artery disease: combination with echocardiography or scintigraphy, or both? $\mathcal{F}$ Am Coll Cardiol 1993;22 159-67.

6 The Veterans Administration Coronary Artery Bypass Surgery Co-operative Study Group. Eleven-year survival in the Veterans Administration randomised trial of coronary bypass surgery in stable angina. $N$ Engl $\mathcal{F}$ Med 1984;11: 1333-9.

7 European Coronary Surgery Study Group. Long term results of prospective randomised study of coronary artery results of prospective randomised study of coronary artery
bypass surgery in stable angina pectoris. Lancet 1982;ii: bypass sur.

8 CASS Principal investigators and their Associates. Coronary Artery Surgery Study (CASS): a randomised trial of coronary artery bypass surgery; survival data. Circulation 1983;68:939-50.

9 Sawada SG, Segar DS, Ryan T, et al. Echocardiographic detection of coronary artery disease during dobutamine infusion. Circulation 1991;83:1605-14.

10 Segar DS, Brown SE, Sawada SG, et al. Dobutamine stress echocardiography: correlation with coronary lesion severity as determined by quantitative angiography. $f \mathrm{Am}$ Coll Cardiol 1992;19:1197-202.

11 Mazeika PK, Nadazdin A, Oakley CM. Dobutamine stress echocardiography for detection and assessment of coronary artery disease. F Am Coll Cardiol 1992;19:1203-11.

12 Pennell DJ, Underwood SR, Swanton RH, et al. Dobutamine thallium myocardial perfusion tomography. $\mathcal{f} \mathrm{Am}$ Coll Cardiol 1991;18:1471-9.

13 Marwick T, Willemart B, D'Hondt A, et al. Selection of the optimal non-exercise stress for the evaluation of ischaemic regional myocardial dysfunction and malperfusion: comparison of dobutamine and adenosine using echocardiogra- phy and Tc-99m-MIBI single photon emission computed omography. Circulation 1993;87:345-54

14 Dennis CA, Pool PE, Perrins EJ, et al. Stress testing with closed-loop arbutamine as an alternative to exercise. $f \mathrm{Am}$ Coll Cardiol 1995;26:1151-8.

15 Cohen JL, Ottenweller JE, George AK, et al. Comparison of dobutamine and exercise echocardiography for detecting coronary artery disease. Am f Cardiol 1993;72:1226-31.

16 Cohen JL, Chan KL, Jaarsma W, et al. Arbutamine echocardiography: efficacy and safety of a new pharmacoogic stress agent to induce myocardial ischaemia and detect coronary artery disease. F Am Coll Cardiol 1995;26: $1168-75$

17 Kiat H, Iskandrian AS, Villegas BJ, et al. Arbutamine stress thallium-201 single photon emission computed tomography using a closed loop delivery system-multicenter trial for evaluation of safety and diagnostic accuracy. $₹ \mathrm{Am}$ Coll Cardiol 1995;26:1159-67.

18 Braunwald E, ed. Heart disease: a textbook of cardiovascular medicine, 4th ed Philadelphia: WB Saunders, 1992:168-9.

19 Senior R, Glenville B, Basu S, et al. Dobutamine echocardiography and thallium-201 imaging predict functional mprovement in severe ischaemic left ventricular dysfunction. Br Heart $\mathcal{F}$ 1995;74:358-64

20 Senior R, Basu S, Handler C, et al. Diagnostic accuracy of dobutamine stress echocardiography for detection of coronary artery disease in hypertensive patients. Eur Heart f 1996; 17:289-95.

21 O'Keefe JH, Baruhart CS, Bateman T. Comparison of stress echocardiography and stress myocardial perfusion scintigery disease and assessing its severity. Am f Cardiol 1995; 75:25-34D.

22 Galanti G, Sciagra R, Comeghio M, et al. Diagnostic accuracy of peak exercise echocardiography in coronary artery disease: comparison with thallium-201 myocardial scintigraphy. Am Heart 7 1991;122:1609-16.

23 Hecht HS, DeBord L, Shaw R, et al. Supine bicycle stress echocardiography versus tomographic thallium-201 exercise imaging for the detection of coronary artery disease. $\mathcal{F}$ Am Soc Echocardiog 1993;6:177-85.

24 Maddahi J, Abdulla A, Garcia EV, et al. Non-invasive idenification of left main and triple vessel coronary artery disease: improved accuracy using quantitative analysis of regional myocardial stress distribution and washout of thallium-201. F Am Coll Cardiol 1986;7:53-60.

25 McCarthy DM, Sciacca RR, Blood DK, et al. Discriminate function analysis using thallium-201 scintigraphy and exercise stress test variables to predict the presence and extent of coronary artery disease. Am F Cardiol 1982;49;1917-26.

26 Hung J, Chaitman BR, Lam J, et al. A logistic regression analysis of multiple noninvasive tests for the prediction of the presence and extent of coronary artery disease in men. Am Heart F 1985;110:460-9.

27 Christian TF, Miller TD, Bailey KR, et al. Noninvasive identification of severe coronary artery disease using exercise tomographic thallium-201 imaging. $A m$ f Cardiol 1992;70:14-20.

28 Nygaard TW, Gibson RS, Ryan JM, et al. Prevalence of high-risk thallium-201 scintigraphic findings in left main coronary artery stenosis: comparison with patients with multiple and single-vessel coronary artery disease. $\mathrm{Am} \mathcal{F}$ Cardiol 1984;53:462-9.

29 Dash H, Massie M, Botvinick EH, et al. The noninvasive identification of left main and 3-vessel coronary artery disease by myocardial stress perfusion scintigraphy and treadmill exercise electrocardiography. Circulation 1979;60:27684

30 Pollock SG, Abbot RD, Boucher CA, et al. A model to predict multivessel coronary artery disease from the exercise thallium-201 stress test. Am f Med 1991;90:345-52.

31 Roger VL, Pellikka PA, Oh JK, et al. Identification of multivessel coronary artery disease by exercise echocardiography. $\mathcal{F}$ Am Coll Cardiol 1994;24:109-14.

32 Quinones MA, Verani MS, Haichin RM, et al. Exercise echocardiography versus Tl-201 single-photon emission computed tomography in evaluation of coronary artery disease. Analysis of 292 patients. Circulation 1992;85:102631

33 Verzijilbergen JF, Suthorp MJ, Asroop CA, et al. Combined assessment of technetium-99m sestamibi planar myocardial perfusion images at rest and during exercise with rest/ exercise left ventricular wall motion studies evaluated from gated myocardial perfusion studies. Am Heart 7 1992;123: $59-68$.

34 Kaul S, Senior R, Dittrich H, et al. Detection of coronary artery disease using myocardial contrast echocardiography: comparison with Tc-99m sestamibi single photon emission computed tomography. Circulation 1997;96:785-92.

35 Davidson CJ, Sheikh KH, Kisslo KB, et al. Intracoronary ultrasound evaluation of interventional technologies. $A m \mathcal{F}$ Cardiol 1991;68:1305-9. 